\title{
cAMP-independent effects of GLP-1 on $\beta$ cells
}

\author{
Jelena Kolic and Patrick E. MacDonald \\ Department of Pharmacology and Alberta Diabetes Institute, University of Alberta, Edmonton, Alberta, Canada.
}

\begin{abstract}
The ability of glucose to stimulate insulin secretion from the pancreatic islets of Langerhans is enhanced by the intestinal hormone glucagon-like peptide 1 (GLP-1), which is secreted from the gut in response to nutrient ingestion. This action, called the incretin effect, accounts for as much as half of the postprandial insulin response and is exploited therapeutically for diabetes treatment through the use of incretin mimetic drugs and inhibitors of dipeptidyl peptidase 4, which degrades GLP-1. Despite a prominent role for incretin mimetics in diabetes treatment, several key questions remain about CLP-1-induced insulin secretion. Most studies have examined the effects of GLP-1 at concentrations several orders of magnitude higher than those found in vivo; therefore, one might question the physiological (and perhaps even pharmacological) relevance of pathways identified in these studies and whether other important mechanisms might have been obscured. In this issue of the $J \mathrm{Cl}$, Shigeto and colleagues demonstrate that physiological CLP-1 does indeed amplify the insulin secretory response. Intriguingly, while much of this response is PKA dependent, as might be expected, the use of picomolar CLP-1 reveals a new and important mechanism that contributes to GLP-1-induced insulin secretion.
\end{abstract}

\section{GLP-1 amplification of glucose-} stimulated insulin secretion

It is well established that the incretin hormone glucagon-like peptide 1 (GLP-1) amplifies the insulin secretory response to glucose $(1,2)$. Specifically, nutrient sensing in the gut promotes intestinal release of GLP-1, which, in turn, enhances insulin secretion by pancreatic $\beta$ cells (3-5). It is generally agreed that GLP-1 receptor (GLP-1R) signaling involves activation of adenylyl cyclase and increased cAMP generation, which, in turn, leads to cAMP-dependent activation of the PKA and exchange protein directly activated by cAMP (EPAC) pathways that promote insulin secretion in a glucose-dependent manner $(1,6)$. However, bioactive GLP-1 has a very short half-life in plasma due to rapid degradation by dipeptidyl peptidase 4 (DPP-4), and thus postprandial plasma levels remain in the picomolar range (7,
8). Even following therapeutic administration of a DPP-4 inhibitor, the circulating concentration of GLP-1 is only increased 2-fold (9). This modest increase in circulating GLP-1 contrasts greatly with the majority of in vitro studies of GLP-1 action on the pancreatic islet, including our own $(11,12)$, which relies on nanomolar concentrations of GLP-1 (or the DPP-4-resistant analog exendin-4).

This discrepancy between the concentration of circulating GLP-1 and the concentrations that have been used to stimulate insulin secretion in vitro raises questions about how GLP-1-dependent actions promote insulin release by pancreatic $\beta$ cells. The reported observations of direct GLP-1 actions on islet function may not be physiologically relevant, and alternative hypotheses have been advanced that suggest that the actions of GLP-1 involve a neuronal gut-to-brain-to-

\section{Related Article: p. 4714}

Conflict of interest: Patrick E. MacDonald has received research funding from Pfizer within the last fiscal year. Reference information: / Clin Invest. 2015;125(12):4327-4330. doi:10.1172/JCI85004. periphery axis. Proponents of these pathways argue that L-cell-secreted GLP-1 influences brain neuronal activities via the vagus nerve, and the brain, in turn, transmits signals to the pancreas to regulate glucose homeostasis $(12,13)$. While accumulating evidence supports the contribution of extrapancreatic GLP-1Rs, other studies continue to strengthen the notion that GLP-1R activation directly in the pancreas is sufficient for incretin control of insulin release (14). However, the question remains as to how physiological concentrations of GLP- 1 act on $\beta$ cells to promote insulin secretion and whether this mechanism has any overlap with the "classical" mechanism(s) defined by studies using supraphysiological GLP-1.

\section{A direct effect}

In this issue, Shigeto and colleagues (15) analyzed human and murine pancreatic islets and show that picomolar concentrations of GLP-1 do indeed augment insulin secretion through direct interaction with the islet GLP-1R. Consistent with previous studies $(16,17)$, Shigeto et al. confirm that GLP-1 action in pancreatic $\beta$ cells is PKA dependent, as pharmacological PKA inhibitors reduced the insulinotropic effect. This block was only partial, suggesting a substantial PKA-independent contribution to the amplification of insulin secretion by GLP-1. Previous work has suggested that GLP-1R signaling is coupled to the activation of PLC (18), and Shigeto et al. show that the PKA-independent action of picomolar concentrations of GLP-1 is mediated by the stimulation of PLC and activation of a depolarizing $\mathrm{Na}^{+}$conductance, which is mediated, in part, by the transient receptor potential (TRP) family of channels. The authors have directly demonstrated that TRPM4 and TRPM5 channels mediate this $\mathrm{Na}^{+}$ conductance in response to GLP-1R activation. Specifically, pharmacological inhibition or knockout of either TRPM4 or TRPM5 suppressed GLP-1-induced action potential firing and insulin secretion in isolated islets. 
A
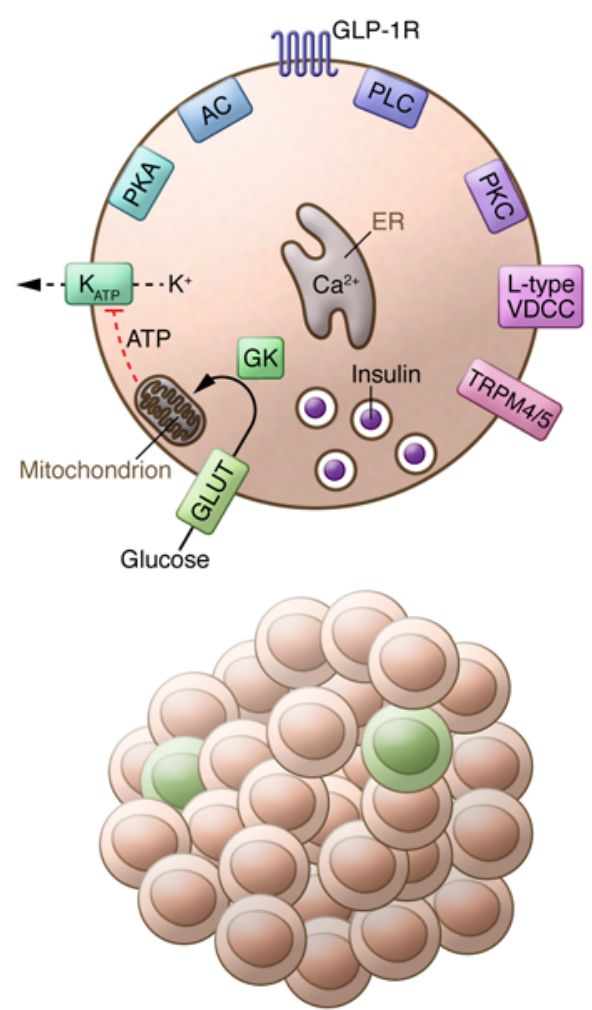

Nonresponsive $\beta$ cell
B
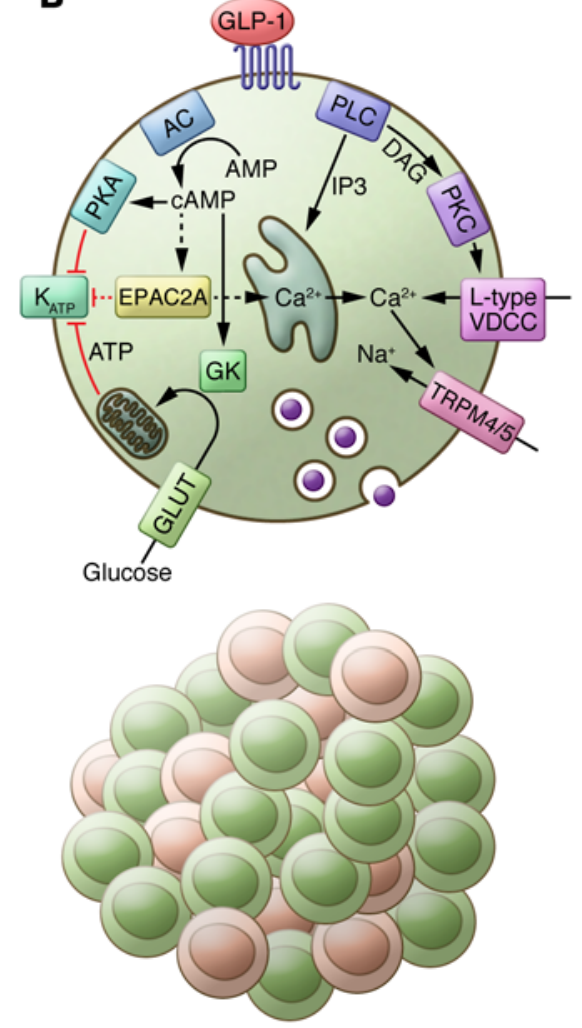

Electrically responsive $\beta$ cell
C
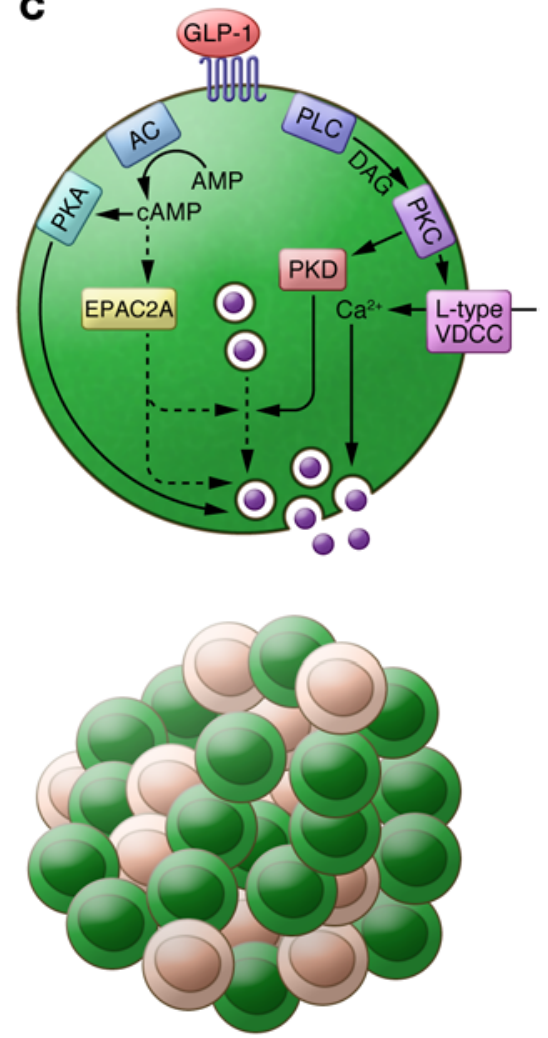

Responsive $\beta$ cell with amplified exocytosis

Insulin secreted

Figure 1. Multiple insulinotropic targets of GLP-1 have been implicated by in vitro studies with physiological and supraphysiological GLP-1. (A) At or near the threshold concentration ( $\sim 6 \mathrm{mM}$ ) for glucose-stimulated insulin secretion, the glucose-dependent inhibition of $\mathrm{K}_{\mathrm{ATP}}$ channels is only sufficient to trigger electrical and $\mathrm{Ca}^{2+}$ responses in a minority of $\beta$ cells. (B) GLP-1 increases the number of responsive $\beta$ cells through PKA- and PLC-dependent pathways, including the PLC-dependent pathway that activates TRPM4 and TRPM5, as shown by Shigeto et al. (15). (C) Within electrically active $\beta$ cells, GLP-1 amplifies the amount of insulin secreted by increasing insulin granule recruitment/docking and by enhancing the exocytotic response to $\mathrm{Ca}^{2+}$ through the PKA-dependent phosphorylation of exocytotic proteins and actin remodeling, the latter of which is possibly mediated by PKD. Although Shigeto et al. suggest that CAMP may not rise sufficiently with picomolar amounts of GLP-1 to promote EPAC2A activation, a role for multiple EPAC2A targets cannot be ruled out. Likewise, further work will be required to determine whether the PKA-dependent component is activated (perhaps locally) by picomolar GLP-1 or is permissive for insulin secretion under this condition. AC, adenylyl cyclase; DAC, diacylglycerol; GK, glucokinase; GLUT, glucose transporter; VDCC, voltage-dependent $\mathrm{Ca}^{2+}$ channels.

In addition, Shigeto and colleagues examined the mechanism that links GLP$1 \mathrm{R}$ to activation of TRPM4 and TRPM5 channels (15). Direct measurements of diacylglycerol production and PKC target phosphorylation and the use of selective PKC inhibitors confirmed the activation and involvement of the PLC pathway. While TRPM4 and TRPM5 channels are directly activated by $\mathrm{Ca}^{2+}$, there are several possible routes by which GLP-1 can act to raise $\left[\mathrm{Ca}^{2+}\right]_{\mathrm{i}}$ in the $\beta$ cell (19). For example, the rise in $\left[\mathrm{Ca}^{2+}\right]_{i}$ can be sourced from direct influx of extracellular $\mathrm{Ca}^{2+}$ or through intracellular release from either the ER or acidic lysosome-related granule stores. Shigeto et al. show that picomolar amounts of GLP- 1 increase $\left[\mathrm{Ca}^{2+}\right]_{\mathrm{i}}$ largely through inositol triphosphate-dependent ( $\mathrm{IP}_{3}$-dependent) mobilization of $\mathrm{Ca}^{2+}$ from the ER (15). Use of the noncompetitive inhibitor of the sarco-ER $\mathrm{Ca}^{2+}$ ATPase (SERCA) thapsigargin prevented the GLP1-mediated $\left[\mathrm{Ca}^{2+}\right]_{\mathrm{i}}$ increase; however, a role for $\mathrm{Ca}^{2+}$ released from acidic granule stores via activation of two-pore channel 1 (TPC1) and TPC2 by nicotinic acid adenine dinucleotide phosphate (NAADP) (20) can not be entirely ruled out. Nonetheless, it seems most likely that picomolar concentrations of GLP-1 act, in part, through the PLC-dependent release of $\mathrm{ER} \mathrm{Ca}^{2+}$ stores to activate TRPM4 and TRPM5 channels and thus promote $\beta$ cell depolarization and electrical activity.

\section{Combined effects enhance $\beta$ cell responses to glucose}

How do the PLC and classical cAMP pathways work together to amplify insulin secretion? A glucose-dependent insulinotropic effect is a defining feature of 
GLP-1 and critical to the advent of incretin mimetics and DPP-4 inhibitors for diabetes therapy. A mechanism whereby picomolar concentrations of GLP-1 alone are sufficient to promote $\beta$ cell depolarization and action potential firing seems poorly aligned with the glucose dependence of GLP-1 action, as action potential firing should stimulate, rather than amplify, a secretory response. Critically, the experiments performed by Shigeto et al. (15) were largely performed with a concentration of glucose $(6 \mathrm{mM})$ that is near the threshold for stimulating insulin secretion (Figure 1A); therefore, the addition of picomolar concentrations GLP-1, which activated depolarizing TRPM4 and TRPM5 channels and inhibits hyperpolarizing $\mathrm{K}_{\mathrm{ATP}}$ channels (e.g., ref. 21), can effectively "sensitize" $\beta$ cells and increase activity at threshold glucose concentrations. These effects, combined with activation of glucokinase (22), facilitation of $\mathrm{Ca}^{2+}$ channel activity (as shown by Shigeto et al. and elsewhere; i.e., ref. 23), and inhibition of voltage-gated $\mathrm{K}^{+}$ channels (10), likely underlie the ability of GLP-1 to confer "glucose competence" to $\beta$ cells (ref. 24 and Figure 1B). Thus, by studying physiological concentrations of GLP-1, Shigeto et al. have identified an important component of the GLP-1-sensitive machinery that effectively recruits more $\beta$ cells within an islet to respond to glucose stimulation.

While activation of TRPM4 and TRPM5 channels can increase the proportion of $\beta$ cells that respond to a glucose stimulus, this mechanism alone cannot make $\beta$ cells secrete more insulin on a cell-by-cell basis. One or more additional mechanism(s) must work to augment the secretory response (Figure 1C). While elucidating a role for PLC-dependent regulation of TRPM4 and TRPM5 channels in GLP-1-mediated effects on insulin secretion, Shigeto et al. confirmed that PKA signaling and direct facilitation of the $\beta$ cell exocytotic response are important for amplifying the insulin secretory response at physiological GLP-1 concentrations (15). Based on these and other recent mechanistic insights, it seems likely that PKA directly augments insulin granule priming and responsiveness to $\mathrm{Ca}^{2+}(25$, 26). Together, these studies indicate that GLP- 1 increases the number of $\beta$ cells that respond to glucose (through mechanisms that include the PLC-dependent activation of TRPM4 and TRPM5 identified by Shigeto et al.) and enhances the secretory capacity of each $\beta$ cell in a PKA-dependent manner downstream of electrical excitability and $\mathrm{Ca}^{2+}$ entry (Figure 1).

\section{Conclusions and future directions}

The fact that Shigeto et al. (15) observed little or no cAMP and PKA activation directly in response to picomolar amounts of GLP-1 is somewhat perplexing. There are two possible scenarios to explain this apparent lack of cAMP/PKA activation: (a) localized $\mathrm{cAMP} / \mathrm{PKA}$ signals were missed or obscured in the context of whole-cell measurements or, (b) at picomolar GLP-1 concentrations, PKA is not stimulated per se but instead plays a permissive role. These scenarios can also be applied to EPAC2A, which has long been implicated in GLP-1-induced insulin secretion. While Shigeto and colleagues suggest that the low levels of cAMP likely to be induced by picomolar concentrations of GLP-1 preclude involvement of EPAC in insulin secretion under this condition, this possibility remains uninvestigated and cannot be ruled out. Clearly, further investigation of the classical cAMP/PKA/EPAC pathways is required, and future studies should examine direct coupling to potential targets or use new and improved tools to measure cAMP/PKA/EPAC activity in $\beta$ cells in response to physiological levels of GLP-1. For example, a more effective PKA antagonist with enhanced membrane permeability (Rp-8-pCPT-cAMPS-pAB) has recently been described (27) and used to demonstrate a role for PKA activity, in the absence of exogenous cAMP-raising agents, in biphasic glucose-stimulated insulin secretion (27). Application of this tool should also provide additional insight into the relative contribution of cAMP-dependent and -independent effects of GLP-1R signaling. Additionally, it would be interesting to examine the effect of Rp-8-pCPT-cAMPS$\mathrm{pAB}$ on the insulinotropic response to picomolar GLP-1 concentrations.

Taken together, the evidence from Shigeto et al. (15) contributes fresh information that provides added insight into the still-emerging network of insulinotropic actions and targets of GLP- 1 in the $\beta$ cell (Figure 1). Collectively, these studies indi- cate that GLP-1 enhances glucose-stimulated insulin secretion by (a) recruiting an increased proportion of $\beta$ cells to respond to glucose through increased excitability mediated by GLP-1 effects on metabolism and ion channels - effects that are both PKA-dependent and -independent (23) - and (b) promoting responsive $\beta$ cells to secrete more insulin on a cell-by-cell basis through increased access of secretory granules to the plasma membrane (11) and the PKA-dependent phosphorylation of exocytotic proteins $(25,26)$, leading to increased exocytotic responses. Importantly, Shigeto and colleagues provide evidence to support a direct insulinotropic effect of physiologically relevant concentrations of the GLP- 1 hormone on the $\beta$ cell. Their use of picomolar concentrations of GLP-1 to evoke membrane depolarization and increase insulin secretion reveals a heretofore unappreciated role for the $\mathrm{G} \alpha_{\mathrm{q}}$-dependent activation of PLC, PKC, and TRPM4 and TRPM5 channels, which likely play an important role in the GLP-1dependent recruitment of glucose-responsive $\beta$ cells.

\section{Acknowledgments}

Work in the MacDonald lab on incretin receptor signaling is funded by an operating grant from the Canadian Diabetes Association (OG-3-14-4565-PM to P.E. MacDonald). P.E. MacDonald holds a Canada Research Chair in Islet Biology.

Address correspondence to: Patrick MacDonald, University of Alberta, Alberta Diabetes Institute, LKS Centre, Rm. 6-126, Edmonton, Alberta, Canada, T6G 2E1. Phone: 780.492.8063; E-mail: pmacdonald@ualberta.ca.

1. Drucker DJ, Philippe J, Mojsov S, Chick WL, Habener JF. Glucagon-like peptide I stimulates insulin gene expression and increases cyclic AMP levels in a rat islet cell line. Proc Natl Acad Sci U S A. 1987;84(10):3434-3438.

2. Mojsov S, Weir GC, Habener JF. Insulinotropin: glucagon-like peptide I (7-37) co-encoded in the glucagon gene is a potent stimulator of insulin release in the perfused rat pancreas. JClin Invest. 1987;79(2):616-619.

3. Perley MJ, Kipnis DM. Plasma insulin responses to oral and intravenous glucose: studies in normal and diabetic subjects. JClin Invest. 1967;46(12):1954-1962.

4. Thorens B. Expression cloning of the pancreatic beta cell receptor for the gluco-incretin hormone glucagon-like peptide 1. Proc Natl Acad Sci US A. 
1992;89(18):8641-8645.

5. Weir GC, Mojsov S, Hendrick GK, Habener JF. Glucagonlike peptide I (7-37) actions on endocrine pancreas. Diabetes. 1989;38(3):338-342.

6. Kashima Y, et al. Critical role of cAMP-GEFII Rim 2 complex in incretin-potentiated insulin secretion. J Biol Chem. 2001; 276(49):46046-46053.

7. Mentlein R, Gallwitz B, Schmidt WE. Dipeptidylpeptidase IV hydrolyses gastric inhibitory polypeptide, glucagon-like peptide-1(7-36)amide, peptide histidine methionine and is responsible for their degradation in human serum. Eur J Biochem. 1993;214(3):829-835.

8. Orskov C, Wettergren A, Holst JJ. Secretion of the incretin hormones glucagon-like peptide-1 and gastric inhibitory polypeptide correlates with insulin secretion in normal man throughout the day. Scand J Gastroenterol. 1996;31(7):665-670

9. Herman GA, et al. Effect of single oral doses of sitagliptin, a dipeptidyl peptidase- 4 inhibitor, on incretin and plasma glucose levels after an oral glucose tolerance test in patients with type 2 diabetes. JClin Endocrinol Metab. 2006;91(11):4612-4619.

10. MacDonald PE, et al. Antagonism of rat betacell voltage-dependent $\mathrm{K}^{+}$currents by exen$\operatorname{din} 4$ requires dual activation of the cAMP/ protein kinase A and phosphatidylinositol 3-kinase signaling pathways. J Biol Chem. 2003;278(52):52446-52453.

11. Kolic J, Spigelman AF, Smith AM, Manning Fox JE, MacDonald PE. Insulin secretion induced by glucose-dependent insulinotropic polypeptide requires phosphatidylinositol 3-kinase $\gamma$ in rodent and human $\beta$-cells. JBiol Chem. 2014;289(46):32109-32120.

12. Sandoval D, Cota D, Seeley RJ. The integrative role of CNS fuel-sensing mechanisms in energy balance and glucose regulation. Annu Rev Physiol. 2008;70:513-535.

13. Cabou C, Burcelin R. GLP-1, the gut-brain, and brain-periphery axes. Rev Diabet Stud. 2011;8(3):418-431.

14. Lamont BJ, et al. Pancreatic GLP-1 receptor activation is sufficient for incretin control of glucose metabolism in mice. JClin Invest. 2012;122(1):388-402

15. Shigeto M, et al. GLP-1 stimulates insulin secretion by PKC-dependent TRPM4 and TRPM5 activation. J Clin Invest. 2015;125(12):4714-4728.

16. Lester LB, Langeberg LK, Scott JD. Anchoring of protein kinase A facilitates hormone-mediated insulin secretion. Proc Natl Acad Sci US A. 1997;94(26):14942-14947.

17. Wang X, Zhou J, Doyle ME, Egan JM. Glucagonlike peptide-1 causes pancreatic duodenal homeobox-1 protein translocation from the cytoplasm to the nucleus of pancreatic $\beta$-cells by a cyclic adenosine monophosphate/protein kinase A-dependent mechanism. Endocrinology. 2001;142(5):1820-1827.

18. Dillon JS, et al. Cloning and functional expression of the human glucagon-like peptide-1 (GLP-1) receptor. Endocrinology.1993;133(4):1907-1910.
19. Rorsman P, Braun M, Zhang Q. Regulation of calcium in pancreatic $\alpha$ - and $\beta$-cells in health and disease. Cell Calcium. 2012;51(3-4):300-308.

20. Zhao Y, Graeff R, Lee HC. Roles of cADPR and NAADP in pancreatic cells. Acta Biochim Biophys Sin (Shanghai). 2012;44(9):719-729.

21. Suga $S$, et al. cAMP-independent decrease of ATP-sensitive $\mathrm{K}^{+}$channel activity by GLP-1 in rat pancreatic $\beta$-cells. Pflugers Arch. 2000;440(4):566-572.

22. Ding SY, et al. Glucagon-like peptide 1 stimulates post-translational activation of glucokinase in pancreatic beta cells. J Biol Chem. 2011;286(19):16768-16774.

23. MacDonald PE, et al. The multiple actions of GLP-1 on the process of glucose-stimulated insulin secretion. Diabetes. 2002; 51(suppl 3):S434-S442.

24. Holz GG, Kühtreiber WM, Habener JF. Pancreatic $\beta$-cells are rendered glucose-competent by the insulinotropic hormone glucagon-like peptide-1(7-37). Nature. 1993;361(6410):362-365.

25 . Song WJ, et al. Snapin mediates incretin action and augments glucose-dependent insulin secretion. Cell Metab. 2011;13(3):308-319.

26. Wu B, et al. Synaptotagmin-7 phosphorylation mediates GLP-1-dependent potentiation of insulin secretion from $\beta$-cells. Proc Natl Acad Sci U S A. 2015;112(32):9996-10001.

27. Schwede F, et al. Rp-cAMPS Prodrugs reveal the cAMP dependence of first-phase glucosestimulated insulin secretion. Mol Endocrinol. 2015;29(7):988-1005 